\title{
Spatio-temporal distribution of malaria one year after the implementation of additional preventive strategies in Bandiagara, Mali
}

Drissa Coulibaly ( $\sim$ coulibalyd@icermali.org )

Malaria Research and Training Center https://orcid.org/0000-0002-7412-0940

Boureima Guindo

Malaria Research and Training Center

Fayçal Maiga

Malaria Research and Training Center

Salimata Konate

Malaria Research and Training Center

Astou Diallo

Malaria Research and Training Center

Abdou Tahirou Mohamed Antar

Malaria Research and Training Center

Amadou Niangaly

Malaria Research and Training Center

Abdoulaye Kassoum Kone

Malaria Research and Training Center

Karim Traore

Malaria Research and Training Center

Matthew B Laurens

Malaria Research Program, Center for Vaccine Development and Global Health, University of Maryland

School of Medicine

Ogobara K Doumbo

Malaria Research and Training Center

Mahamadou Ali Thera

Malaria Research and Training Center

\section{Research}

Keywords: Malaria, time-series, parasite carriage, mapping, spatial distribution

Posted Date: November 4th, 2020 
DOI: https://doi.org/10.21203/rs.3.rs-100617/v1

License: (c) (1) This work is licensed under a Creative Commons Attribution 4.0 International License. Read Full License 


\section{Abstract}

\section{Background}

Evaluation of local transmission epidemiology to characterize malaria risk is essential for planning malaria control and elimination programmes. The use of geographical information systems (GIS) has been a major asset to this approach. This study aimed to characterize the local spatio-temporal pattern of malaria infection and clinical disease after implementation of Seasonal Malaria Chemoprevention (SMC) and Indoor Residual Spraying (IRS) in Bandiagara, a Malian town.

\section{Methods}

From October 2017 to December 2018, an active and passive surveillance system was established in a cohort study of three hundred children aged from 6 months to 15 years old. Weekly time-series of clinical malaria and monthly time-series of asymptomatic Plasmodium carriage and rainfall were plotted. Numbers of clinical malaria episodes and asymptomatic parasite carriers were mapped using Quantum Geographic Information System (QGIS). Landscape features of Bandiagara were obtained from Google earth. Clusters of high or low risk were identified under SaTScan ${ }^{\circledR}$ software using a Bernoulli model.

\section{Results}

From October 2017 to December 2018, 167 clinical malaria cases were recorded, mostly from July to December, while asymptomatic parasite carriage was observed during the entire study period. Three clusters of clinical episodes were found. All were hotspots. They were located in the north-east, south and west. No low risk cluster was identified. Three significant high-risk clusters of asymptomatic parasite carriage were identified in the south, north-east and north. These clusters were located near standing water.

\section{Conclusion}

This study confirms the seasonality of clinical malaria in Bandiagara. The continued presence of asymptomatic parasite carriers maintains malaria transmission. To advance malaria elimination, control strategies must also target hotspots of asymptomatic parasite carriage. There was a spatial heterogeneity of clinical and asymptomatic malaria. Despite the implementation of additional preventives strategies, the locations of high-risk clusters were stable.

\section{Background}

Malaria is the most dangerous and widespread parasitic disease in the world. Currently, nearly half of the world's population lives in malaria-endemic areas and is at risk of contracting malaria. According to the World Health Organization (WHO), 3.2 billion people live in malaria endemic areas. Two hundred and twenty-eight million (228 million) clinical episodes and 405,000 deaths from malaria were recorded in 2018 (1). 
Malaria is mainly transmitted by anopheline bites, which represent the vector. The abundance of vectors depends on environmental factors such breeding sites. The association of malaria transmission with specific locations is attributable to the presence of anopheline vector breeding sites. Each breeding site can be the center of a focus of malaria transmission (2). In Africa, transmission levels vary enormously and transmission can be either seasonal or throughout the year (3). Differences exist not only between different regions, but also at the local level (4). The factors determining local transmission intensity include vector profile, ecology and seasonality; all of these items have an impact on the effectiveness of control operations (5). Local variations should be considered when considering the risk of infection.

A decrease in malaria incidence has been reported in various places in Africa following the implementation of control strategies that impact transmission (6). The burden of Plasmodium falciparum is characterized by spatial and temporal variability that presents new and evolving challenges for malaria control programs. Reductions in malaria burden need to be sustained in the face of changing epidemiology whilst simultaneously tackling significant pockets of sustained or increasing transmission (6).

As the transmission decline the transmission of disease is becoming more and more focal(7) and the epidemiology changes (8), pockets of transmission, or 'hot spots', characterize low transmission areas and must be identified for an area to be declared malaria-free (9). The location of incident malaria cases identified through a passive surveillance system may be indicative of asymptomatic malaria reservoirs (10).

Spatial heterogeneity, spatial dynamics, and seasonality are of great interest for spatial and seasonal targeting, which could enable tailoring interventions and coverage targets to the local context and identifying hotspots $(11,12)$.

Characterizing the spatial variation of disease prevalence by mapping exercises has proven useful for strategic planning of malaria prevention and control activities at the national level (13-15).

In Mali, the Seasonal Malaria Chemoprevention (SMC) targeting children from 3-59 months was scaled up in 2016. In 2017 and 2018, Indoor Residual Spraying (IRS) was implemented in few health districts including Bandiagara. Previous studies showed a marked spatial heterogeneity of malaria transmission in Bandiagara $(16,17)$. This study aimed to characterize the local spatio-temporal features of clinical and asymptomatic malaria in the recent context of SMC and local IRS implementation.

\section{Method}

\section{Study setting}

The study was done in Bandiagara town, situated in central Mali in West Africa. A detailed description of the study site can be found elsewhere (17). Children aged from 3-59 months residing in Bandiagara benefited from SMC since 2016. IRS was implemented in all areas of Bandiagara town in 2017 and 2018. 


\section{Cohort description}

A prospective cohort study interspersed with monthly cross-sectional surveys involving three hundred children was conducted from October 2017 to December 2018.

The study inclusion criteria were: aged between 6 months and 15 years at the time of screening, resident of Bandiagara town, good general health based on clinical evaluation, written informed consent obtained from the parent/guardian, and the availability in follow-up for the entire study duration. Exclusion criteria were: refusal to participate in the study, simultaneous participation in an interventional clinical trial, chronic medication with known anti-malarial activity (such as trimethoprim-sulfamethoxazole for prevention of AIDS-associated opportunistic infections), and any condition that in the opinion of the principal investigator would jeopardize the safety or rights of a participant in the trial or would render the participant unable to comply with the protocol.

Active and passive surveillance were conducted to capture the incidence of malaria infection and disease. Active surveillance consisted of scheduled monthly visits aimed at detecting asymptomatic malaria infection and anaemia. Clinical examination of the participants was performed by the study physician at enrolment and on a quarterly basis. Following standard protocols, finger-prick blood samples were collected monthly for malaria smears, measurement of haemoglobin level, and parasite genotyping from filter paper. Venous blood was collected at enrolment and at the time of each malaria clinical episode for molecular and immunological analyses.

Passive surveillance consisted of continuous availability of medical care at the Bandiagara Malaria Project (BMP) research clinic and Bandiagara District Hospital, where parents/guardians were instructed to consult whenever their child was sick. Children were then examined by a physician, and axillary temperature was checked (fever was defined as axillary temperature $\geq 37.5^{\circ} \mathrm{C}$ ). Blood samples were collected for microscopic examination for malaria parasites (thick blood film). If malaria was confirmed by microscopy, the haemoglobin level was determined, and filter paper collected for parasite genotyping. Clinical malaria was defined by the presence of one of the following symptoms consistent with malaria: fever (subjective or measured), headache, nausea or vomiting, diarrhea or abdominal pain, join pain, chills, seizure, lethargy, coma associated with a positive Plasmodium asexual stage.

Asymptomatic malaria was defined by the presence of Plasmodium irrespective of the stage without the symptoms listed above.

Clinical malaria cases were treated with artemether + lumefantrine combination therapy according to the Malian National Malaria Control program (NMCP) guidelines.

\section{Malaria and rainfall time-series}

After aggregation on a weekly time scale, time series of the number of malaria episodes was plotted together with rainfalls measured at the local weather station in Bandiagara. 
For asymptomatic malaria time-series, the monthly cumulated number of asymptomatic cases and measured rainfalls were plotted together.

Rainfall data were obtained from the local weather station. Microsoft Excel version 2016 was used for plotting time-series.

\section{Case mapping and spatial clusters detection}

At enrollment, each child's household (i.e., the place where the child slept) was georeferenced using a tablet with Open Data Kit (ODK) platform (accuracy approximately within $10 \mathrm{~m}$ ).

A Geographical Information System was developed for the study area that also included the Bandiagara house blocks and bodies of water in the area.

Google earth was used for digitization of road infrastructure and water collection, and the administrative boundaries of Bandiagara town. After digitization, data were exported to QGIS and ARC GIS software for map processing. Data from Google Earth Pro, which was in Keyhole Markup Language (KML) file format was converted to Shapefile (SHP) to facilitate georeferencing in the geographic coordinate system.

Using Quantum GIS ${ }^{T M}$ software (QGIS ${ }^{T M}$ ) version 2.18.1, participant households were mapped according to their geographic coordinates. Numerous children, likely siblings of the same family, shared the same location. For each location, data were subsequently aggregated, and several variables were calculated: initial number of study participants, total number of recorded malaria episodes or symptomatic malaria cases. The spatial distribution of malaria risk was illustrated by choropleth mapping of the number of malaria episodes at the block level.

SaTScan ${ }^{\mathrm{TM}}$ software, version 9.6, using the Kulldorf method of retrospective space-time permutation and the Bernoulli purely spatial model was used to determine the spatial variability of malaria risk, a cluster analysis was performed using Kulldorff's statistics through the SaTScan ${ }^{\mathrm{TM}}$ software version 9.6 with Poisson model SaTScan ${ }^{\mathrm{TM}}$ version $9.6(18,19)$. This widely applied method $(20,21)$ moves a circular or elliptic scanning window over the study area and compares observed and expected case numbers inside and outside this window in order to detect clusters and estimate risk ratios. Using daily malaria episodes at each location, a Bernoulli distribution model with $50 \%$ of the population at risk, and elliptic scanning windows, high or low risk purely spatial clusters were sought over the study period. The Relative-risk was defined as the ratio of observed to expected cases. Cluster significance ( $P$-value) was computed with a likelihood ratio test provided by a Monte Carlo approach using 999 random simulations under the null hypothesis of no cluster. Statistically significant spatial clusters $(P$-value $<0.05)$ were subsequently mapped on QGIS ${ }^{\mathrm{TM}}$.

\section{Ethical compliance}

The study was conducted in compliance with the International Conference on Harmonization Good Clinical Practices, the Declaration of Helsinki and regulatory requirements of Mali. Details on the consent 
and the protocol approval process have been described elsewhere ${ }^{17}$. The study was approved by the institutional ethics committee of the Faculty of Medicine dentistry and pharmacy of the University of Sciences Techniques and Technologies of Mali.

\section{Results}

\section{Clinical malaria episodes and rainfall time-series}

To analyse the temporal distribution of malaria episodes, data were aggregated by week, and a time series of malaria episodes was plotted together with locally measured rainfalls. At the beginning of the study (17 October 2017) which corresponds to the 42nd week, the rains stopped. The last clinical malaria case of 2017 was diagnosed during the 48th week (27 November 2017), (Figure1).

In 2018 the rains began at the week 21 (24 May 2018). In the last two weeks of July, no rain was measured, and clinical malaria episodes started being recorded (2018W39) (Figure1). Rains were frequent from 31st week to the 39th week (2018W31-2018W39) which cover the month of August. The peak incidence of clinical malaria episodes was observed during the $39^{\text {th }}$ week (last week of September) (Figure1). No rain was measured from 2018W39 to 2018W41. The rainy season stopped at week 42 (14 October) (Figure1). Children continued to experience clinical malaria up to the end of the study (December 2018) (Figure1).

\section{Asymptomatic malaria and rainfall time-series}

During the follow-up period, study participants experienced asymptomatic Plasmodium carriage irrespective of rainy versus dry season. The peak of asymptomatic malaria parasite carriage was observed in May 2018 (Figure 2).

\section{Spatial distribution, case mapping and clusters identification}

Children habitats were mainly patchy distributed (Figure 3). During the study period, 16 active surveys were carried out.

\section{Symptomatic malaria spatial distribution}

During the study, 167 malaria episodes were mapped. Episodes mostly concentrated along the Yamé River and the vicinity of the brickyard located in the North-east (Figure 4).

Three high-risk clusters were identified. The first was located in the north-east (population=44, number of cases $=79, R R=7.00, p<10^{-6}$ ). The second in the south (population=16, number of cases $41, R R=7.64$ $p<10^{-6}$ ). The third in the west (population $=4$, number of cases $10, R R=5.91 p=0.0038$ ).

No low-risk cluster was found. 
Two hundred eighty-two Plasmodium asymptomatic carriers were identified. Three hotspots of asymptomatic malaria were identified. The first covered a large southern part of the town with a population of 66 subjects, 57 number of cases, a RR=3.50, and $p<10^{-6}$. The second hotspot in the northeast with a population of 21 subjects, number of cases $21, R R=3.63, p=0.00058$. The third was located in the north (population 20, number of cases 18, RR=3.23 p=0.013). The last two clusters were not far from the brickyard. No low cluster was found (Figure 5).

\section{Discussion}

A decrease in malaria burden consecutive to the implementation of preventive strategies such us the SMC has been showed in numerous studies (22-26).

This study describes a fine scale space and time profile of malaria after immediate recent implementation of SMC and IRS in a malaria research site and sentinel site of NMCP.

Time-series profile

On the intra-annual scale, seasonal periodicity in rainfall and temperature leads to seasonal fluctuation in vector populations, parasite development rates and malaria transmission.

Our study started as the rainy stopped. Only nine clinical malaria episodes were recorded from the beginning of the study (October 2017) up to June 2018. Usually the peak of transmission is in September and/or October depending of the rainfall pattern. The low number of clinical episodes may be due to the implementation of malaria preventive strategies: universal coverage of Long-Lasting Insecticide Treated Nets (LLITN), IRS and SMC. The study spanned the entire year of 2018, numbers of malaria clinical episodes were recorded. Clinical malaria incidence in our study was 0.5 episodes per person year (published elsewhere) which was low compared to the period preceding SMC and the IRS implementation. The incidence of clinical malaria in participants aged from 3 months to 20 years was 1.7 episodes per 24 weeks from 1999-2000 and 1.4 episodes person year in children aged from 6 months to 14 years old from $2009-2013(27,28)$. These findings confirm the seasonality of clinical malaria.

Seasonality in malaria transmission is driven by temporal fluctuations in rainfall and temperature, which determine the ability of the environment to support development of mosquitoes $(29,30)$.

Asymptomatic Plasmodium carriers represent a parasite reservoir. They were found during the entire study follow-up period irrespective of season. The permanent presence of asymptomatic carriers leads to sustainable malaria transmission. To advance malaria elimination, these carriers must to targeted by control measures. To our knowledge, in sub-Saharan Africa no particular control strategy is directed to this population. This may explain why despite enthusiastic reduction of malaria burden, transmission is maintained. 
The peak of asymptomatic malaria parasite carriage was observed in May 2018 just before the beginning of the malaria transmission season. Asymptomatic parasite carriers represent a bottle neck for control programs. The prevalence of asymptomatic carriage may be underestimated because microscopy was used for diagnosis, while PCR is known to be more sensitive (31) .

Spatial distribution profile

Maps provide a powerful visual tool to identify areas where targeted strategies and resources are most likely to have the greatest impact.

Heterogeneity in the spatial distribution of malaria transmission, at increasingly localized scales, was illustrated in South Africa (32).

The clinical malaria episodes in our study mostly concentrated along the Yamé River and the vicinity of the brickyard located in the north-east, and hotspots were located in the north-east, south and western sides of the town. Hotspots were located close to the river Yamé and the brickyard represent the same hotspots identified before SMC and IRS implementation $(16,17)$.

The hotspots of asymptomatic carriers were located in the south, north and north-east. The existence of river Yamé and the brickyard that keeps standing water from the rainy season up to March constitute mosquito breeding sites that remain after the rains have ended.

Hotspots represent an opportunity for targeted control interventions that are expected to be more efficient than untargeted interventions and ultimately benefit the whole community (7).

Limitations of this study are the lack of assessment of entomological and environmental factors that may impact the surveys.

\section{Conclusion}

This study confirmed the seasonality and fine scale heterogeneity of malaria risk.

The permanent presence of asymptomatic parasite carriers supports the sustainability of the transmission. To substantially decrease malaria transmission and advance elimination, control strategies must target the hotspots, especially for asymptomatic Plasmodium carriers. Despite the implementation of SMC and IRS, the locations of the high-risk clusters remained stable.

\section{Abbreviations}

GIS

Geographic Information Systems

SMC

Seasonal Malaria Chemoprevention 
IRS

Indoor Residual Spray

LLINs

Long-lasting insecticide-treated bed nets

NMCP

National Malaria Control Program

MARCAD

Malaria Research Capacity Development

DELTA

Developing Excellence in Leadership, Training and Science in Africa

AAS

African Academy of Sciences

AESA

Alliance for Accelerating Excellence in Science in Africa

NEPAD

New Partnership for Africa's Development Planning and Coordinating Agency.

\section{Declarations}

\section{Ethics approval and consent to participate}

The study was approved by the institutional ethics committee of the Faculty of Medicine dentistry and pharmacy of the University of Sciences Techniques and Technologies of Mali.

\section{Consent for publication}

Not applicable.

\section{Availability of data and material}

The datasets used and/or analysed during the current study are available from the corresponding author on reasonable request.

\section{Competing interests}

The authors declare no conflicts of interest.

\section{Funding}

This work was supported through the DELTAS Africa Initiative grant DEL-15-10. The DELTAS Africa Initiative is an independent funding scheme of the African Academy of Sciences (AAS)'s Alliance for Accelerating Excellence in Science in Africa (AESA) and supported by the New Partnership for Africa's Development Planning and Coordinating Agency (NEPAD Agency), with funding from the Wellcome Trust 
grant $107741 / A / 15 / Z$ and the UK government. The views expressed in this publication are those of the authors (s) and not necessarily those of AAS, NEPAD Agency, Wellcome Trust or the UK government.

\section{Authors' contributions}

DC, MAT and OKD were involved in the conceptualization, research design, data collection and preparation of the manuscript. $B G, F M, A K K, K T, A D, A N$ contributed significantly to study execution and data collection. $\mathrm{M} T$ and $\mathrm{MBL}$ reviewed the manuscript. All authors read and approved the final manuscript.

\section{Acknowledgements}

The authors are grateful to the Malaria Research Capacity Development (MARCAD) programme, part of the Developing Excellence in Leadership, Training and Science in Africa (DELTA) consortium. The views expressed in this publication are those of the author (s) and not necessarily those of AAS, NEPAD Agency, Wellcome Trust or the UK government. The content is solely the responsibility of the authors and does not necessarily represent the official views of the National Institutes of Health.

We thank Ibrahima Kebe and Ismael Thera for data management; Abdoualye Ongoiba, Kadidia Coulibaly and Lassine Sanankoua for mapping support; Boucari Djiguiba, Abdoulaye Ouologuem, Sadio Dolo, Domo Ouologuem, Issa Tapily, and Paul Dougnon for fieldwork; Danzélé Coulibaly, and Sékouba Mariko, for administrative and technical support; Aboubacar Doumbia, the medical chief officer of the Bandiagara District Hospital, for support; and the community of Bandiagara, Mali for their dedication.

\section{References}

1. World malaria report. 2019 [Internet]. [cited 2020 May 11]. Available from: https://www.who.int/publications-detail/world-malaria-report-2019.

2. Carter R, Mendis KN, Roberts D. Spatial targeting of interventions against malaria. Bulletin of the World Health Organization. 2000;11.

3. O'Meara WP, Bejon P, Mwangi TW, Okiro EA, Peshu N, Snow RW, et al. Effect of a fall in malaria transmission on morbidity and mortality in Kilifi, Kenya. The Lancet. 2008 Nov;372(9649):1555-62.

4. Guerra CA, Gikandi PW, Tatem AJ, Noor AM, Smith DL, Hay SI, et al The Limits and Intensity of Plasmodium falciparum Transmission: Implications for Malaria Control and Elimination Worldwide. Rogerson SJ, editor. PLoS Med. 2008 Feb 26;5(2):e38.

5. Guthmann JP, Llanos-Cuentas A, Palacios A, Hall AJ. Environmental factors as determinants of malaria risk. A descriptive study on the northern coast of Peru. Trop Med Int Health. 2002 Jun;7(6):518-25.

6. Nkumama IN, O'Meara WP, Osier FHA. Changes in Malaria Epidemiology in Africa and New Challenges for Elimination. Trends in Parasitology. 2017 Feb;33(2):128-40. 
7. Bousema T, Griffin JT, Sauerwein RW, Smith DL, Churcher TS, Takken W, et al. Hitting Hotspots: Spatial Targeting of Malaria for Control and Elimination. PLOS Medicine. 2012 Jan 31;9(1):e1001165.

8. Cotter C, Sturrock HJW, Hsiang MS, Liu J, Phillips AA, Hwang J, et al. The changing epidemiology of malaria elimination: new strategies for new challenges. Lancet. 2013 Sep;7(9895):900-11. 382(.

9. Hay SI, Smith DL, Snow RW. Measuring malaria endemicity from intense to interrupted transmission. The Lancet infectious diseases. 2008 Jun;8(6):369.

10. Stresman GH, Kamanga A, Moono P, Hamapumbu H, Mharakurwa S, Kobayashi T, et al. A method of active case detection to target reservoirs of asymptomatic malaria and gametocyte carriers in a rural area in Southern Province, Zambia. Malaria Journal. 2010 Oct;4(1):265. 9(.

11. Reiner RC Jr, Le Menach A, Kunene S, Ntshalintshali N, Hsiang MS, Perkins TA, et al Mapping residual transmission for malaria elimination. Jit M, editor. eLife. 2015 Dec 29;4:e09520.

12. Ruktanonchai NW, DeLeenheer P, Tatem AJ, Alegana VA, Caughlin TT, zu Erbach-Schoenberg E, et al Identifying Malaria Transmission Foci for Elimination Using Human Mobility Data. Koelle K, editor. PLoS Comput Biol. 2016 Apr 4;12(4):e1004846.

13. The effect of malaria control on Plasmodium falciparum in Africa. between 2000 and 2015 | Nature [Internet]. [cited $2020 \mathrm{Jul}$ 30]. Available from: https://www.nature.com/articles/nature15535.

14. A World Malaria Map. Plasmodium falciparum Endemicity in 2007 [Internet]. [cited 2020 Jul 30]. Available from: https://journals.plos.org/plosmedicine/article?id=10.1371/journal.pmed. 1000048 .

15. Noor AM, Kinyoki DK, Mundia CW, Kabaria CW, Mutua JW, Alegana VA, et al. The changing risk of Plasmodium falciparum malaria infection in Africa: 2000-10: a spatial and temporal analysis of transmission intensity. The Lancet. 2014 May;383(9930):1739-47.

16. Coulibaly D, Rebaudet S, Travassos M, Tolo Y, Laurens $M$, Kone AK, et al. Spatio-temporal analysis of malaria within a transmission season in Bandiagara, Mali. Malar J. 2013 Dec;12(1):82.

17. Coulibaly D, Travassos MA, Tolo Y, Laurens MB, Kone AK, Traore K, et al. Spatio-Temporal Dynamics of Asymptomatic Malaria: Bridging the Gap Between Annual Malaria Resurgences in a Sahelian Environment. The American Journal of Tropical Medicine Hygiene. 2017 Dec;6(6):1761-9. 97(.

18. SaTScan_Users_Guide.pdf.

19. Anselin L. Local Indicators of Spatial Association-LISA. Geographical Analysis. 2010 Sep 3;27(2):93-115.

20. Kulldorff M, Heffernan R, Hartman J, Assunção R, Mostashari F. A Space-Time Permutation Scan Statistic for Disease Outbreak Detection. PLOS Medicine. 2005 Feb;15(3):e59. 2(.

21. Generalized Linear Mixed Models Approach for Detecting Incident. Clusters of Disease in Small Areas, with an Application to Biological Terrorism | American Journal of Epidemiology | Oxford Academic [Internet]. [cited $2020 \mathrm{Jul}$ 30]. Available from: https://academic.oup.com/aje/article/159/3/217/79584. 
22. Kokwaro G. Ongoing challenges in the management of malaria. Malaria Journal. 2009 Oct;12(1):2. 8 .

23. Raghavendra K, Barik TK, Reddy BPN, Sharma P, Dash AP. Malaria vector control: from past to future. Parasitol Res. 2011 Apr 1;108(4):757-79.

24. WHO Study Group. Malaria vector control and personal protection. World Health Organ Tech Rep Ser. 2006;936:1-62. back cover.

25. Global Malaria Action Plan -. (Roll Back Malaria, 2008) [Internet]. Child Health Task Force. [cited 2020 Jul 30]. Available from: https://www.childhealthtaskforce.org/resources/action-plan/2008/globalmalaria-action-plan-roll-back-malaria-2008.

26. Gething PW, Battle KE, Bhatt S, Smith DL, Eisele TP, Cibulskis RE, et al. Declining malaria in Africa: improving the measurement of progress. Malar J. 2014 Dec;13(1):39.

27. Coulibaly S, Dicko A, Doumbo OK, Cissoko Y, Thera MA, Lyke K, et al. Impact of preseason treatment on incidence of falciparum malaria and parasite density at a site for testing malaria vaccines in Bandiagara, Mali. The American Journal of Tropical Medicine and Hygiene. 2002 Dec 1;67(6):60410.

28. Coulibaly D, Travassos MA, Kone AK, Tolo Y, Laurens MB, Traore K, et al. Stable malaria incidence despite scaling up control strategies in a malaria vaccine-testing site in Mali. Malar J. 2014;13(1):374.

29. White MT, Griffin JT, Churcher TS, Ferguson NM, Basáñez M-G, Ghani AC. Modelling the impact of vector control interventions on Anopheles gambiae population dynamics. Parasites Vectors. 2011 Jul;28(1):153. 4(.

30. Cairns ME, Walker PGT, Okell LC, Griffin JT, Garske T, Asante KP, et al. Seasonality in malaria transmission: implications for case-management with long-acting artemisinin combination therapy in sub-Saharan Africa. Malar J. 2015 Dec;14(1):321.

31. Adams M, Joshi SN, Mbambo G, Mu AZ, Roemmich SM, Shrestha B, et al. An ultrasensitive reverse transcription polymerase chain reaction assay to detect asymptomatic low-density Plasmodium falciparum and Plasmodium vivax infections in small volume blood samples. Malar J. 2015 Dec;14(1):520.

32. Hay SI, Omumbo JA, Craig MH, Snow RW. Earth observation, geographic information systems and Plasmodium falciparum malaria in sub-Saharan Africa. In: Advances in Parasitology [Internet]. Elsevier; 2000 [cited 2019 Dec 11]. p. 173-215. Available from: https://linkinghub.elsevier.com/retrieve/pii/S0065308X00470090.

\section{Figures}




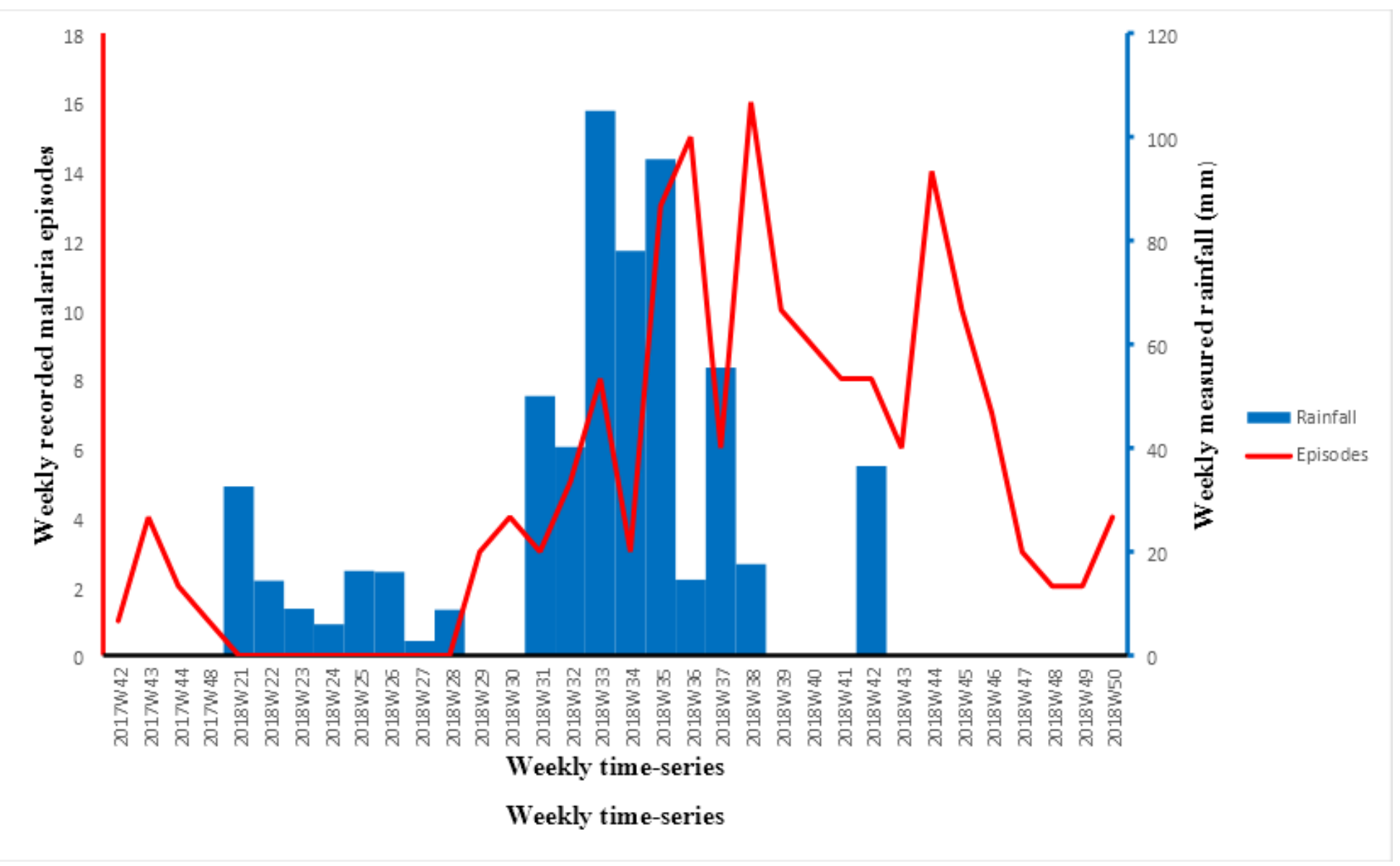

Figure 1

Recorded malaria episodes and measured rainfall weekly time-series 


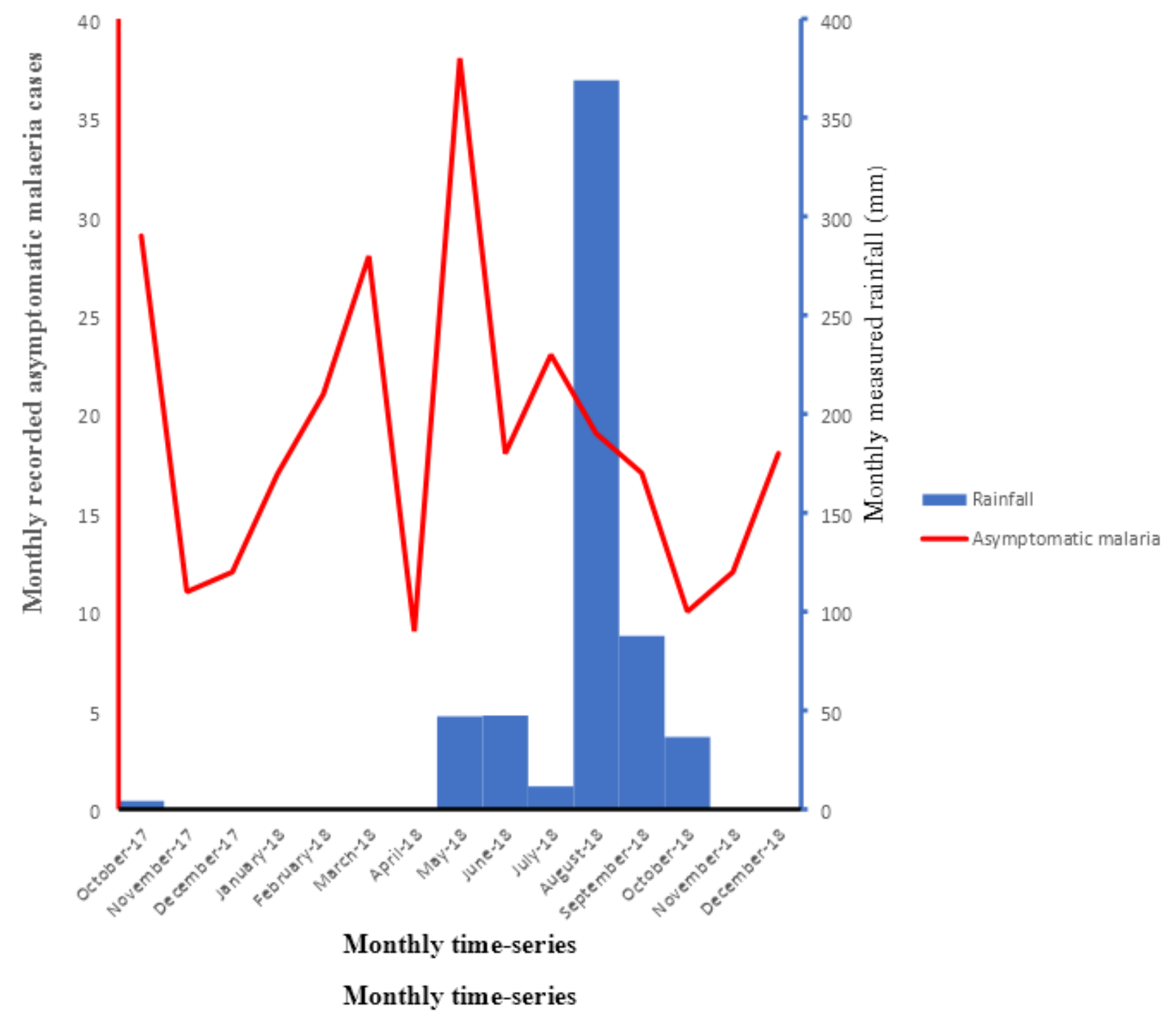

Figure 2

Recorded asymptomatic malaria and measured rainfall monthly time-series 


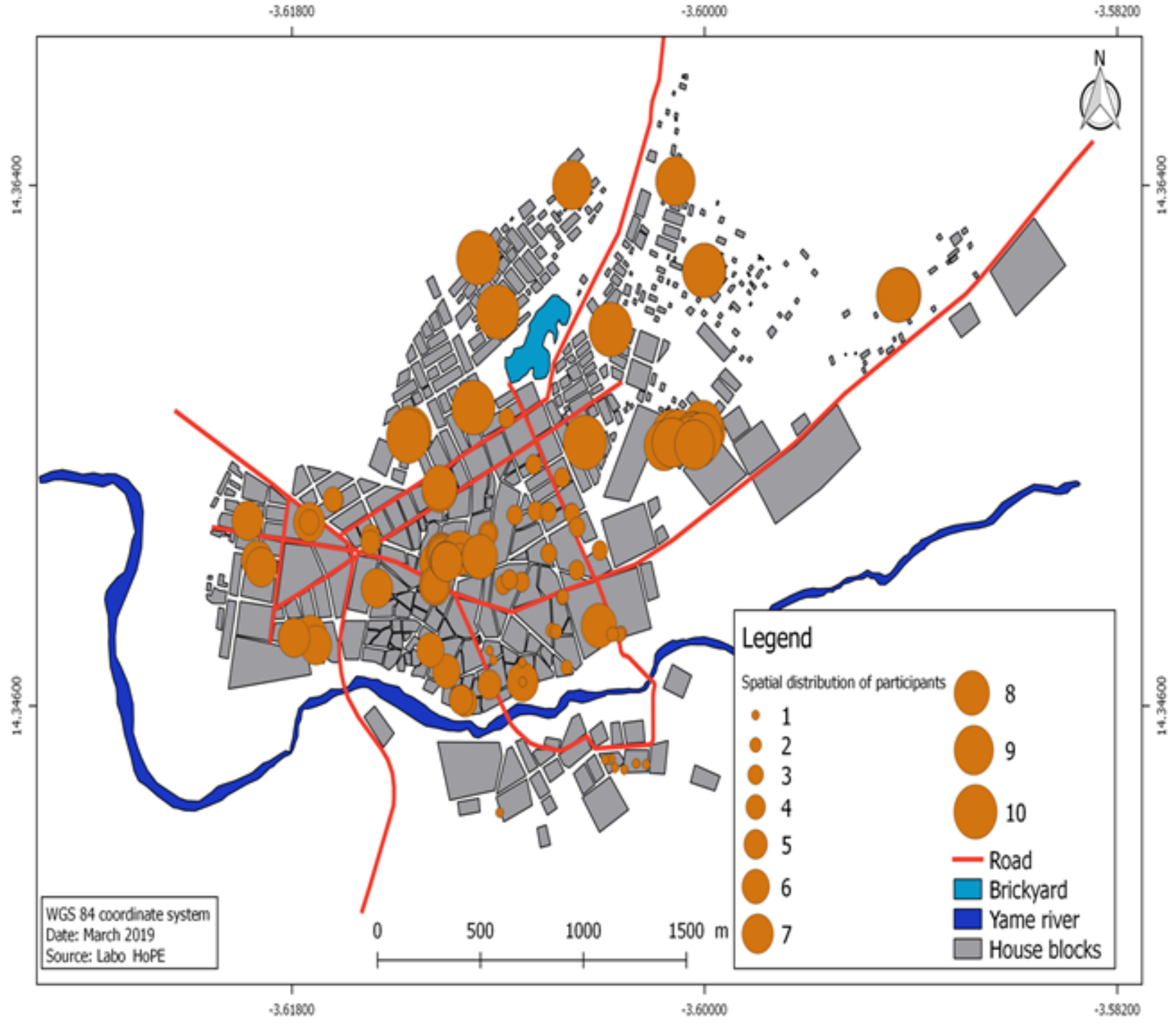

Figure 3

Spatial distribution of the study participants 


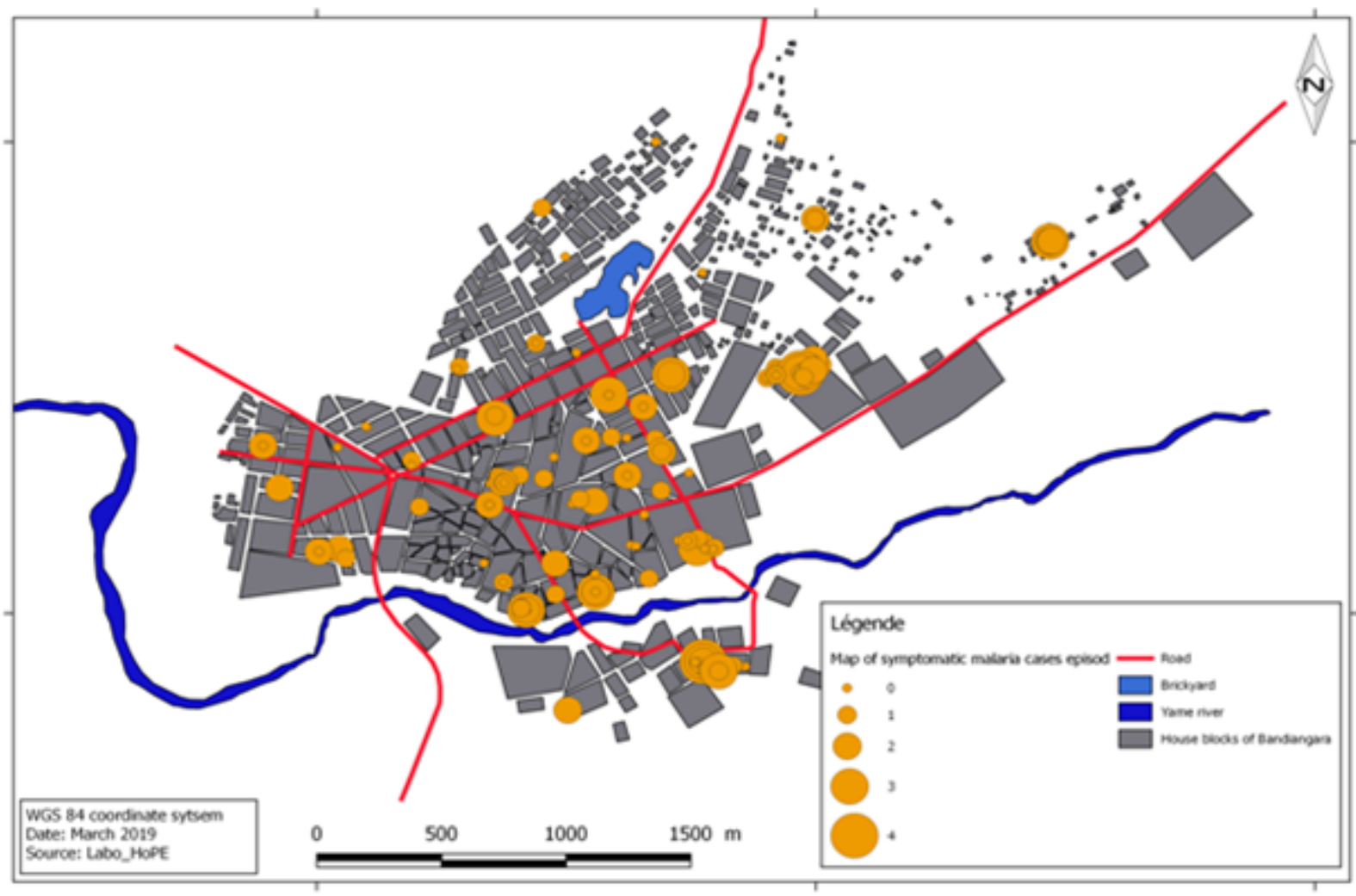

Figure 4

Localization of high-risk clusters of symptomatic malaria computed on SaTScan® 


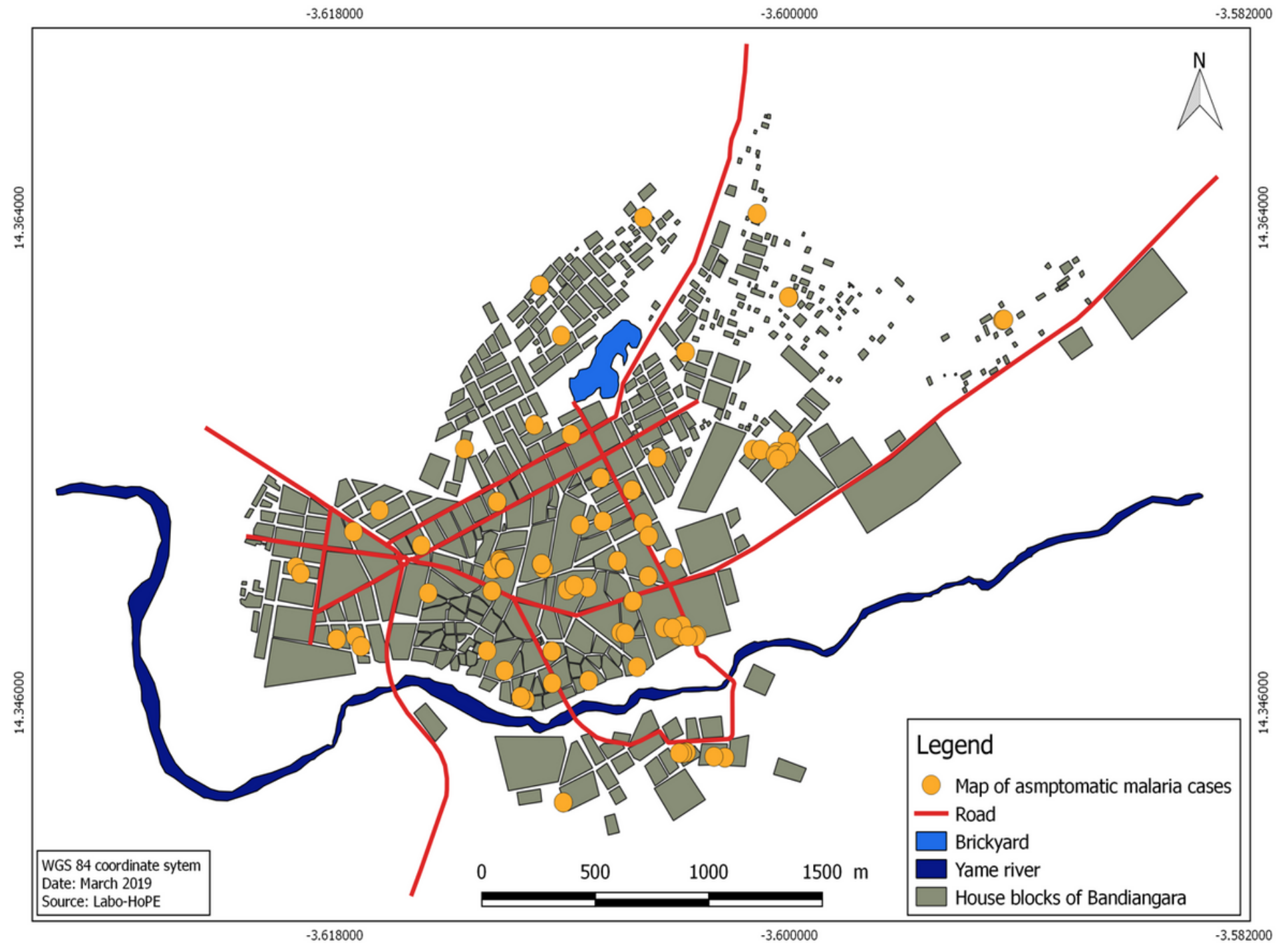

Figure 5

Localization of high-risk clusters of asymptomatic malaria computed on SaTScan ${ }^{\circledR}$ 